\title{
Discrete numerical simulation, quasistatic deformation and the origins of strain in granular materials.
}

\author{
Gaël Combe \& Jean-Noël Roux \\ Laboratoire des Matériaux et des Structures du Génie Civil, Institut Navier, Champs-sur-Marne, France
}

\begin{abstract}
Systematic numerical simulations of model dense granular materials in monotonous, quasistatic deformation reveal the existence of two different régimes. In the first one, the macroscopic strains stem from the deformation of contacts. The motion can be calculated by purely static means, without inertia, stress controlled or strain rate controlled simulations yield identical smooth rheological curves for a same sample. In the second régime, strains are essentially due to instabilities of the contact network, the approach to the limits of large samples and of small strain rates is considerably slower and the material is more sensitive to perturbations. These results are discussed and related to experiments : measurements of elastic moduli with very small strain increments, and slow deformation (creep) under constant stress.
\end{abstract}

\section{INTRODUCTION}

Despite its now widespread use (Kishino 2001), discrete numerical simulation of granular materials, motivated either by the investigation of small scale (close to the grain size) phenomena, or by the study of microscopic origins of known macroscopic laws, still faces difficulties. Microscopic parameters, some of which are to be defined at the (even smaller) scale of the contact, are incompletely known. Macroscopic constitutive laws do not emerge easily out of noisy simulation curves, and the numerically observed $d y$ namic sequences of rearrangements might appear to contradict the traditional macroscopic quasistatic assumption. Detailed and quantitative comparisons with experiments can be used to adjust microscopic models, but a systematic exploration of the effect of the various parameters throughout some admissible range is also worthwhile. This is the purpose of the present study, which also addresses the fundamental issues of the macroscopic and quasistatic limits, in the case of the biaxial compression of dense, two-dimensional (2D) samples of disks.

In section 2, we introduce the model and the numerical methods and define dimensionless parameters that are robust indicators of the relative importance of different phenomena. Rheological curves can be evaluated in the large sample limit (section 3), and their sensitivity to parameters assessed. We observe (section 4) two different mechanical régimes, according to whether the dominant microscopic origin of strain is material deformation in the contacts or rearrangements of the contact network. Connections to some experimental observations are suggested in part 5 , while the conclusion section outlines further perspectives.

\section{NUMERICAL MODEL AND PROCEDURES}

\subsection{Grain-level mechanics}

Our computational procedure is one of the simplest types of 'molecular dynamics' or 'discrete element' method (Cundall and Strack 1979) for solid grains. We consider 2D assemblies of disks, with diameters uniformly distributed between $a / 2$ and $a$, and masses and moments of inertia evaluated accordingly (as for homogeneous solid cylinders of equal lengths). $m$ will denote the mass of a disk of diameter $a$, and $N$ the number of disks.

These grains interact in their contacts with a linear elastic law and Coulomb friction. The normal contact force $F_{N}$ is thus related to the normal deflection (or apparent interpenetration) $h$ of the contact as $F_{N}=K_{N} h Y(h), Y$ being the Heaviside step function (equal to 1 for $h>0$, to 0 otherwise). The tangential component $F_{T}$ of the contact force is proportional to the tangential elastic relative displacement, with a tangential stiffness coefficient $K_{T}$. The Coulomb condition $\left|F_{T}\right| \leq \mu F_{N}$ requires an incremental evaluation of $F_{T}$ every time step, which leads to some amount of slip each time one of the equalities $F_{T}= \pm \mu F_{N}$ is imposed. A normal viscous component opposing the 
relative normal motion of any pair of grains in contact is also added to the elastic force $F_{N}$. Such a term - of unclear physical origin in dense multicontact systems - is often introduced to ease the approach to mechanical equilibrium. Its influence will be assessed in part 3 . The viscous force is proportional to the normal relative velocity, and the damping coefficient in the contact between grains $i$ and $j$ is a constant fraction $\zeta$ $(0 \leq \zeta \leq 1)$ of the critical value $2\left(\frac{K_{N} m_{i} m_{j}}{m_{i}+m_{j}}\right)^{1 / 2}$. (In a binary collision the normal 'restitution coefficient' is 0 for $\zeta=1$ and 1 for $\zeta=0$ ). $\zeta, K_{N}, K_{T}$, and $\mu$ are the same in all contacts. The motion of grains is calculated on solving Newton's equations.

\subsection{Numerical compression tests}

Two different types of boundary conditions are used : either the container walls are physical objects, with masses, satisfying Newton's equations (but requested to move in the direction perpendicular to their orientation), or periodic boundary conditions (no walls) are implemented. In both cases, the changes in cell size and shape under controlled stress involves specific dynamical parameters which could be discussed in more detail. Here we will simply deem such parameter choice innocuous if results are reproducible, sizeindependent and consistent. We use soil mechanics sign conventions for stresses and strains. Samples are first compressed isotropically under a constant pressure $P$. Once a mechanical equilibrium is reached under pressure $P$, samples are submitted to biaxial compression tests. The lateral stress, $\sigma_{1}$ is maintained equal to $P$, while either $\epsilon_{2}$ is increased at a constant rate $\dot{\epsilon}_{2}$ (a procedure hereafter referred to as SRC, for strain rate controlled) or $\sigma_{2}$ is stepwise increased by small fractions of $P$, and one waits for the next equilibrium configuration before changing $\sigma_{2}$ (a SIC, for stress increment controlled, procedure). In the sequel $q$ denotes the ratio $\left(\sigma_{2}-\sigma_{1}\right) / \sigma_{1}$, while $\epsilon_{2}$ and $\epsilon_{v}=\epsilon_{1}+\epsilon_{2}-\epsilon_{1} \epsilon_{2}$ are respectively termed 'axial' and 'volumetric' strain, in analogy with 3D axisymmetrical triaxial tests.

\subsection{Dimensional analysis}

Rheological curves and internal sample states obtained in monotonous biaxial tests are defined in the macroscopic limit $N \rightarrow \infty$. If expressed by relations between dimensionless quantities $\epsilon_{2}, q, \epsilon_{v}$, they should depend on the friction coefficient $\mu$ and on ratio $K_{T} / K_{N}$, and on three other dimensionless parameters: $\kappa=K_{N} / P$, the stiffness parameter, which expresses the level of contact deformation, $\gamma=$ $\dot{\epsilon}_{2} \sqrt{m / P}$, the inertia parameter, evaluating, in SRC (constant $\dot{\epsilon}_{2}$ ) tests, the importance of dynamical effects, and $\zeta$, the damping parameter, introduced in paragraph 2.1, characterizing viscous dissipation. The contact coordination number is a decreasing function of $\kappa$. The quasistatic limit is the limit of small $\gamma$.

\section{BIAXIAL COMPRESSION OF DENSE SYS- TEMS : RESULTS}

\subsection{Preparation, initial states, procedures.}

The sample preparation procedure is well known to exert a strong influence on the mechanical properties of a granular sample as, in particular, dense or loose initial states respond differently (Wood 1990) to load increments. Moreover, experiments also showed that density is not sufficient to determine the behaviour in a triaxial test (Benahmed 2001). Numerical simulations may in principle attempt to imitate as closely as possible laboratory experiments. The simulations of such processes as deposition under gravity within a walled container is however difficult, as it requires large number of particles. Inhomogeneous states one obtains in such cases request samples much larger then a representative volume element, which is itself much larger than the grain size. Moreover, the transition from an initial fluid-like configuration to a solidlike grain assembly is bound to be sensitive to static and dynamic parameters (Silbert et al. 2001).

Here we focus on the slow quasistatic deformation of certain types of granular assemblies, once they have been prepared in some well defined initial state. Therefore we leave a detailed (and necessary) study of the preparation process to future research, and adopt a simple numerical procedure which provides us with homogeneous, reproducible, sample size -independent initial states in equilibrium under an isotropic pressure. The numerical procedure is an isotropic, monotonous compaction from an initial gas-like configuration with a solid fraction $\Phi$ of about $20 \%$. To obtain a dense sample, a different, smaller value is attributed to the coefficient of friction in this initial dynamic compression step. Two series of samples are studied here. The first one - called series A hereafter - was prepared between solid, frictionless walls. It was observed in that case that one had to set $\mu$ to zero in the preparation stage if we were to obtain a homogeneous stress field. Simulations of series A were therefore performed starting from the very dense states which result from a compression without intergranular friction (Combe 2001). The results below, some of which were presented in (Roux and Combe 2002), were obtained with $\mu=0.25$ during biaxial compressions, and a rigidity level $\kappa=10^{5}$. $K_{T} / K_{N}$ was set to $1 / 2$. Biaxial tests were SIC, with small $q$ steps $\delta q=10^{-3}$. Each successive mechanical equilibrium is deemed attained when the total force (or torque) on each grain is less than $10^{-4} a P$ (resp. $10^{-4} a^{2} P$ ) and when the relative difference between the internal overall stresses (deduced from nonviscous intergranular forces) and their prescribed val- 
Table 1: Initial state data for series B simulations.

\begin{tabular}{|l||c|c|c|}
\hline$\kappa$ & $\Phi$ & $z$ & $x_{0}(\%)$ \\
\hline \hline $10^{5}$ & $0.8226 \pm 8.10^{-4}$ & $3.59 \pm 2.10^{-2}$ & $10.0 \pm 0.5$ \\
\hline $10^{4}$ & $0.8230 \pm 8.10^{-4}$ & $3.64 \pm 2.10^{-2}$ & $9.0 \pm 0.6$ \\
\hline $10^{3}$ & $0.8258 \pm 9.10^{-4}$ & $3.77 \pm 8.10^{-3}$ & $6.7 \pm 0.3$ \\
\hline
\end{tabular}

ues is less than $10^{-4}$. $\zeta$ was set to high values (near 1 ) and $N$ ranged from 1024 to 4900 . In the initial isotropic state, the solid fraction (extrapolated to $N \rightarrow \infty$ ) is $\Phi=0.844 \pm 0.001$, all but $5.5 \%$ of the disks carry forces and the coordination number, ignoring those inactive grains, is $z \simeq 4.01$, very close to the isostatic limit (Roux 2000) of 4 reached with rigid, frictionless disks in equilibrium.

For the second series of simulations, series B, we used periodic boundary conditions. Samples are thus devoid of edge effects. They shrink homogeneously in the isotropic compression stage. Series B samples were compressed with $\mu=0.15$, and subsequent biaxial tests performed with $\mu=0.5$. Different stiffness levels, $\left(\kappa=10^{3}, 10^{4}\right.$ and $\left.10^{5}\right)$ were used, with $K_{T} / K_{N}$ fixed to 1 , as well as different inertia parameters $\gamma\left(10^{-3}, 10^{-4}\right.$, sometimes $\left.10^{-5}\right)$. SRC tests were compared to SIC ones (with $\delta q=10^{-2}$ and $\zeta \simeq 1$ ). Samples of 1400 and 5600 disks were simulated. The initial solid fraction, due to the finite $\mu$ value during compression, is lower than for A samples, as well as the coordination number $z$ among force-carrying disks. Values of $\Phi, z$, and the fraction of inactive disks $x_{0}$, for the investigated $\kappa$ values are given in table 11. The typical aspect of $q$ versus $\epsilon_{2}$ curves is illustrated on fig. 1, for series B samples with $\kappa=10^{4}$ and $\gamma=10^{-4}$. They are characteristic of very dense samples, as in (Kuhn 1999).

\subsection{Stress-strain curves and macroscopic limit.}

The increase of $q$ with $\epsilon_{2}$ is initially quite fast, $q$ reaching about 0.8 for $\epsilon_{2}<10^{-3}$. Then the deviator stress keeps increasing and reaches an apparent plateau for $\epsilon_{2} \sim 0.01$. Those dense samples are markedly dilatant (fig. 7 below), after a very small initial contraction their volume steadily increases, even after $q$ appears to have levelled off. The important stress fluctuations in those SRC tests is striking on fig. 1, but are considerably reduced, as well as sample-to-sample differences, as $N$ increases from 1400 to 5600 . Dilatancy curves (see below) are smoother. Smooth stress-strain curves can thus be expected in the macroscopic limit $N \rightarrow \infty$. This was more carefully checked for simulation series $A$, on studying three sample sizes : on fig. 2 the shaded zones extend to one standard deviation on each side of the average curves, for $q$ plotted as a function of $\epsilon_{2}$ for $N=1024$ (26 samples), $N=3025$ (10 samples), and $N=4900$ (7 samples). Fig. 2 does indicate a systematic decrease of the fluc-

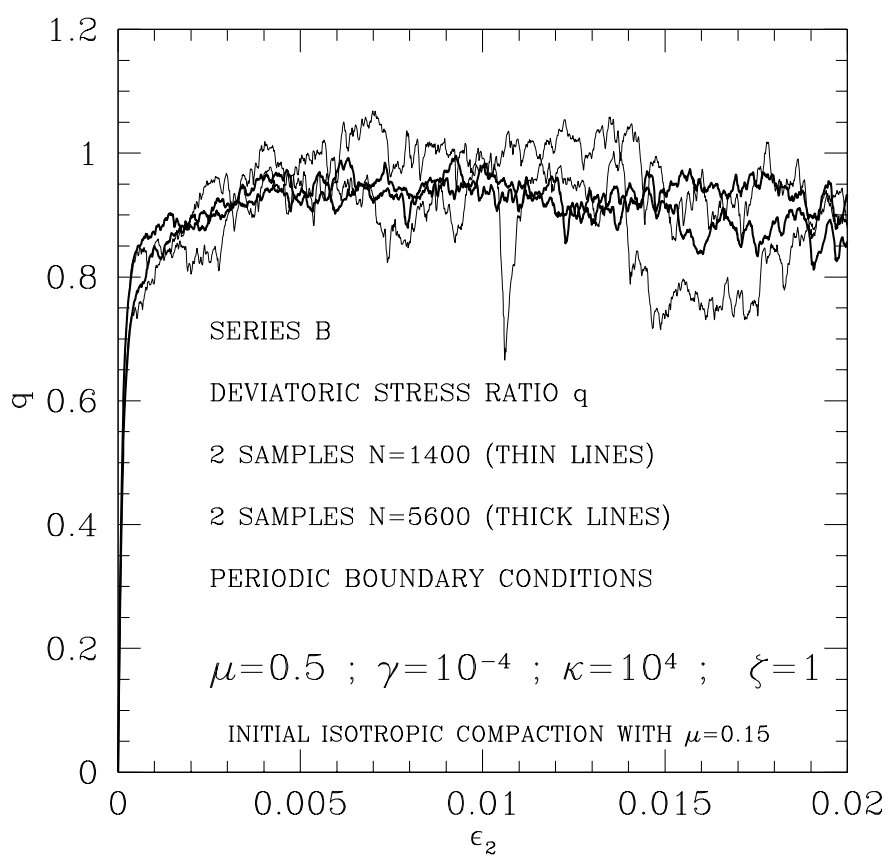

Figure 1: $q$ versus axial strain $\epsilon_{2}$ in B samples of 2 different sizes. Fluctuations are larger for the smaller samples.

tuation level (see inset), compatible with a regression as $N^{-1 / 2}$, just like for an average over a number of independent contributions (subsystems of representative size) proportional to $N$. Series A samples respond in a similar way to deviator stresses as type B ones (although of course, due to different initial states, $\mu$ and $\kappa$, constitutive laws will differ). The initial increase

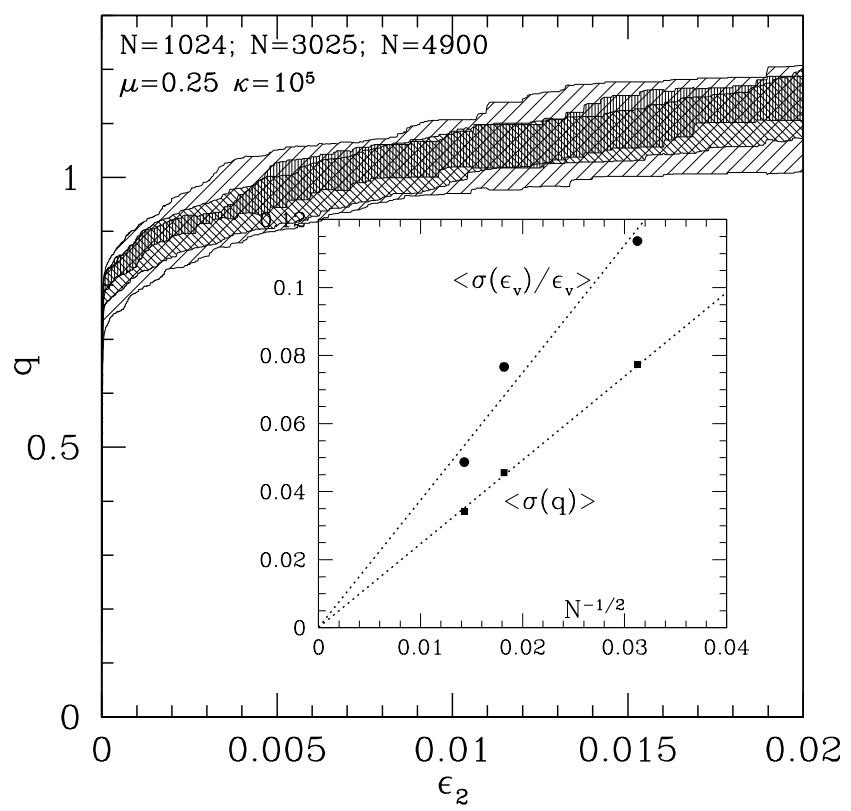

Figure 2: Hashed zone (the darker the larger $N$ ) one r.m.s. deviation on each side of average curve for the 3 sample sizes indicated (series A, SIC with $\delta q=10^{-3}$ ). Inset : its average width over the $\epsilon_{2} \leq 0.02$ interval, versus $1 / \sqrt{N}$, along with the average relative uncertainty on $\epsilon_{v}$ 
of $q$, so fast that it cannot be distinguished from the axis on fig. 2, is followed by a slower variation. (Yet, unlike in the B case, $q$ does not reach a maximum for $\left.\epsilon_{2} \leq 0.02\right)$. 'Volumetric' strains are also qualitatively similar for series A and B.

\subsection{Role of parameters $\zeta, \gamma, \kappa$.}

The quasistatic stress-strain curve should be the same for SRC and SIC biaxial compressions, independent on $\zeta$ and on $\gamma$ if it is small enough. To check this, five samples of series B were submitted to SRC tests with $\gamma=10^{-3}$ and $\zeta=1, \gamma=10^{-4}$ and $\zeta=1, \gamma=10^{-4}$ and $\zeta=0$, and to SIC ones with $\delta q=10^{-2}$. Average curves for $q$ versus $\epsilon_{2}$ (fig. 3) and $\epsilon_{v}$ versus $\epsilon_{2}$ (fig. (4) for those 4 sets of simulations are displayed (and standard deviations levels indicated as on fig. (2). Obviously, the value of $\zeta$ does not have any appreciable influence on the rheological curve. Intergranular friction is the dominating dissipation mechanism, and it can be checked that the differences between stresses evaluated with and without viscous forces differ by negligible amounts for all SRC tests. However, results are affected by the reduced rate $\gamma$, or the choice of an SIC procedure. A smaller $\gamma$ (according to its definition, this amounts to a slower compression, lighter grains or higher pressures) results in smaller deviator and dilatancy values for a given 'axial' strain. SIC tests, as one waits for equilibrium, are the slowest, and SIC curves can be regarded as an extrapolation of SRC ones to $\gamma=0$. (The occurrence of slightly decreasing $q$ values in SIC tests might seem surpris-

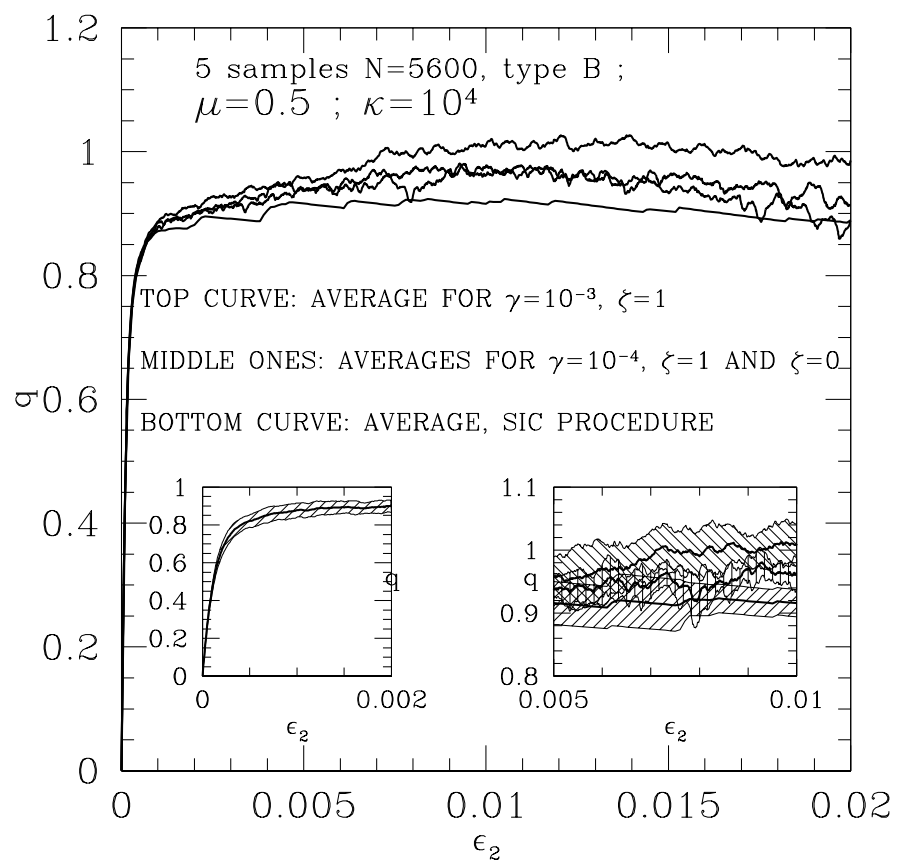

Figure 3: Average $q$ versus $\epsilon_{2}$ for conditions indicated. Left inset: detail of one curve with r.m.s. deviations, small $\epsilon_{2}$. Right inset: averages and r.m.s. deviations for $\gamma=$ $10^{-3}, \gamma=10^{-4}$ and SIC tests.

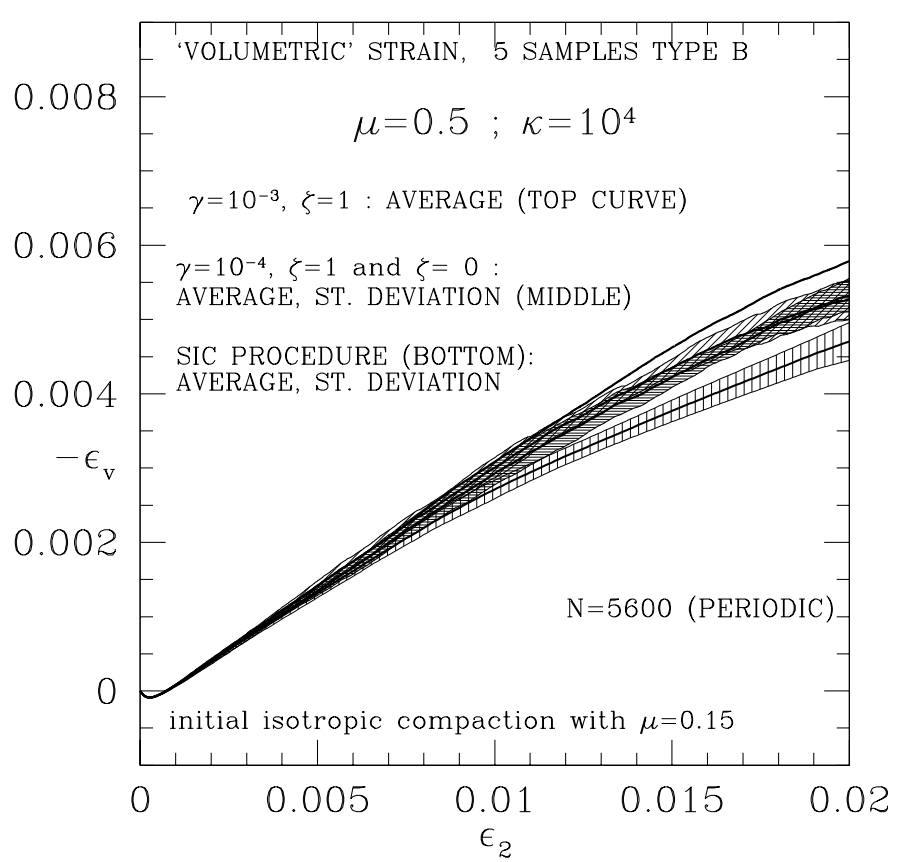

Figure 4: Same as fig. 3 for $\epsilon_{v}$ vs. $\epsilon_{2}$, standard deviations shown except for uppermost $\left(\gamma=10^{-3}\right)$ curve.

ing, but is due to the use of real Cauchy stresses to draw the curve, while stresses defined in terms of initial cell dimensions are used in the calculations). The effects of the stiffness parameter $\kappa$ are illustrated on fig. 5. It is most apparent in the initial rise of $q$, which is the faster for higher $\kappa$, and the small-strain contractant régime (see inset), which develops with softer contacts. For smaller $\kappa$, the packing appears indeed to be softer. The curves at larger strains display no conspicuous difference between $\kappa=10^{4}$ and $\kappa=10^{5}$, although the softest grains, $\kappa=10^{3}$ appear to withstand a somewhat higher deviator stress. The dilatancy - slope of $-\epsilon_{v}$ versus $\epsilon_{2}$ - is not affected. The time scale for stress fluctuation during monotonous tests at a given strain rate is a strongly decreasing function of $\kappa$, hence the smoother curves on fig. 5 for softer contacts. The effects of the parameters on rheological curves are related to some changes in the internal states of the system undergoing compression. The effect of $\gamma$ is related to the greater distance to equilibrium of systems under higher strain rate. Characteristic quantities are the average kinetic energy per particle, $e_{c}$ (in units of $a^{2} P$ ) and the quadratic average of the net force on a particle (in units of $a P$ ), $f_{2}$. Those quantities tend to slowly increase with $\epsilon_{2}$ during the test, but typical values for $\epsilon_{2}=0.01$ can be cited. As for SIC tests, one only records equilibrium positions, ensuring $f_{2} \leq 10^{-5}$ and $e_{c} \leq 10^{-8}$. The coordination number $z$ and the proportion of sliding contacts $X_{s}$ vary quickly before $\epsilon_{2}=10^{-3}$ and remain essentially constant afterwards (one has $z=3.12$, on average, for $\kappa=10^{4}$ and $\gamma=10^{-4}, z=3.05$ for $\kappa=10^{4}$ and $\gamma=10^{-3}$ ). Tests with the highest $\gamma$ val- 
ues $10^{-3}$ are, logically, the farthest from equilibrium $\left(e_{c}=1.510^{-5}\right.$, and $f_{2}=0.01$, while $e_{c} \simeq 510^{-7}$ and $f_{2}=0.02$ for $\gamma=10^{-4}$ ). The change of $\kappa$ makes a significantly larger difference from $10^{4}$ to $10^{3}$ than from $10^{5}$ to $10^{4}$. Unlike $e_{c}$ and $f_{2}$, which essentially depend on $\gamma, z$ and $X_{s}$ are sensitive to both parameters. In SIC tests $\left(\kappa=10^{4}\right), z$ decreases from its initial value to about 3.22 (for $\epsilon_{2} \sim 0.01$ ) which is consistent with its dependence on $\gamma$ in SRC conditions. Intermediate configurations of SIC tests, remarkably, do not have any sliding contact: on approaching equilibrium, all contact forces leave the edge of the Coulomb cone. Upon resuming an SRC motion, very small displacements can mobilize friction and $X_{s}>0$ is observed (typically $X_{s} \simeq 10 \%$, if $\gamma=10^{-4}$ and $\kappa=10^{4}, X_{s}$ increases with $\gamma$ and with $\kappa$ ).

\section{DIFFERENT ORIGINS OF STRAIN}

One striking aspect of the rheological curves is the existence of two different régimes. At small $\epsilon_{2}$, close to the initial isotropic state, curves are quite smooth and reproducible, sample to sample fluctuations are very small (figs. 1] and B), SIC and SRC tests (whatever $\gamma \leq 10^{-3}$ ) are in perfect agreement (figs. 3 and (4), and $\kappa$ strongly affects the results (fig. 5). Coordination numbers and friction mobilization change fast from initial values (table (1) to the roughly constant ones given in paragraph 3.3. At larger strains, the system is sensitive to the strain rate, much more than to the stiffness parameter. Fluctuations are considerably larger, and the stepwise increase of $q$, as one records the ensuing sequence of equilibria, results in a staircase-shaped $q$ versus $\epsilon_{2}$ curve, as on

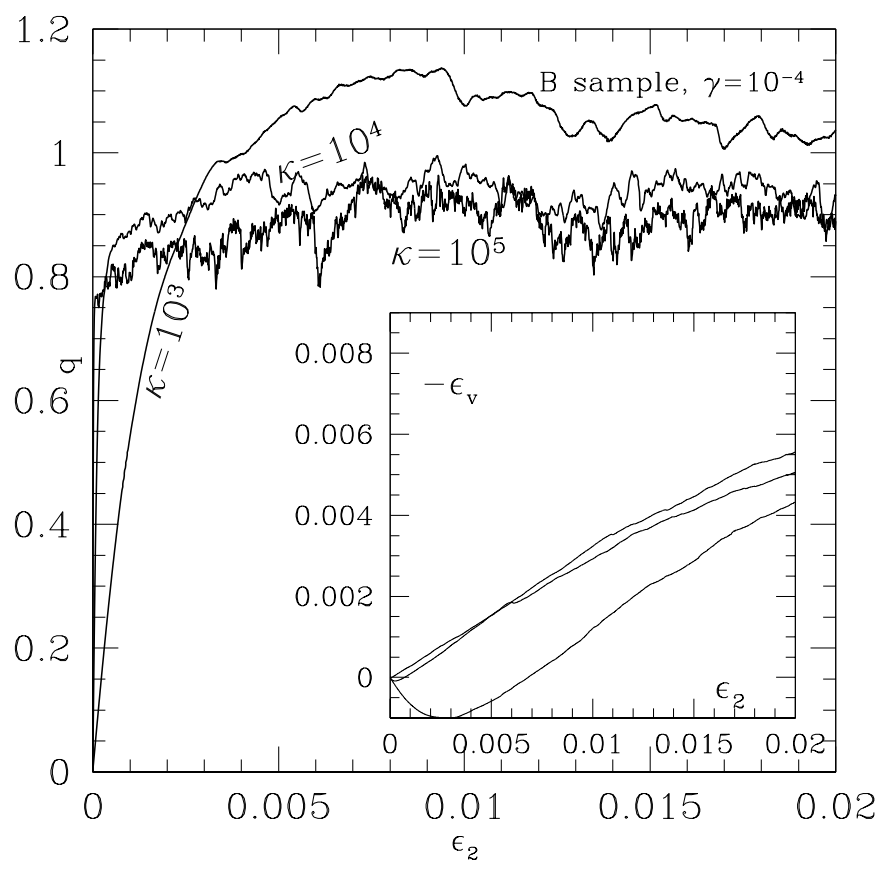

Figure 5: Results for one B-sample with 3 different stiffness values, $q$ (main plot) and $\epsilon_{v}$ (inset) vs. $\epsilon_{2}$.

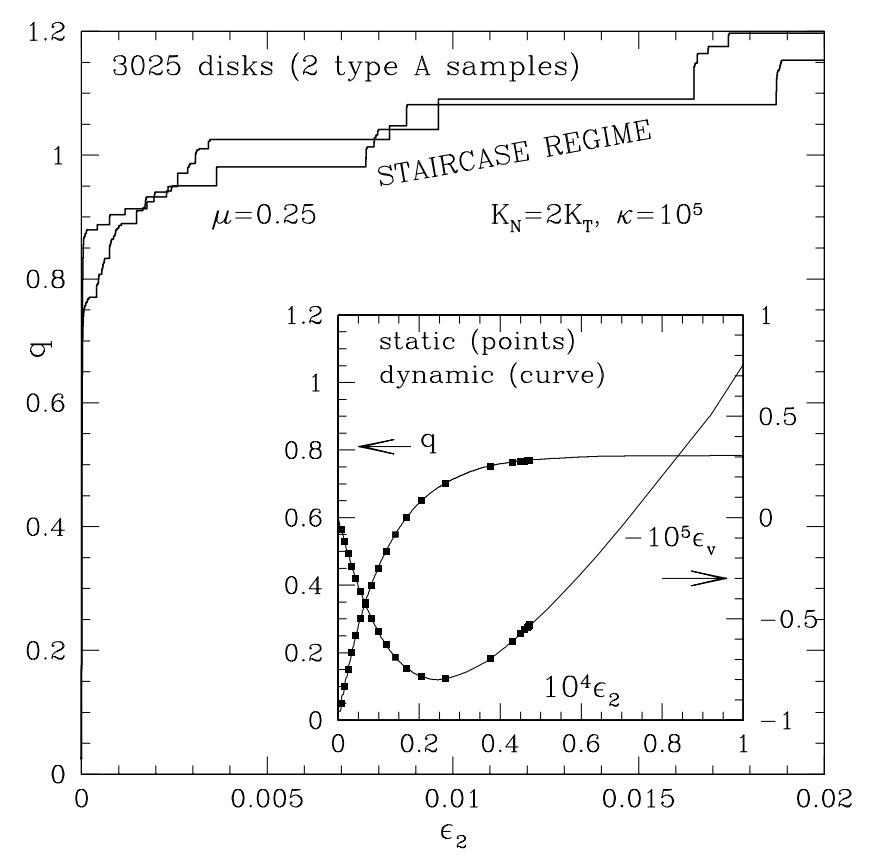

Figure 6: Two SIC $q$ vs. $\epsilon_{2}$ curves. Inset : initial strictly quasistatic régime, blown-up $\epsilon$ scales. Results on one sample are identical with both static and dynamic methods.

fig. 6. $q$ increments in those SIC simulations are very small, $\delta q=10^{-3}$, so that nearly vertical segments on those plots correspond to many different equilibrium configurations, each very close to the previous one. The slope of those steep parts of the curve is close to that of the initial, stiff rise of $q$, confused with the axis on the main plot in the figure, and visible in the blown-up inset. Large horizontal segments are due to motions between more distant configurations. The origin of those two different regimes is clarified once it is attempted to find the system response to small load increments by purely static means. Starting from an equilibrium configuration, it is possible to regard its contact structure as a given network of elastoplastic elements, and determine the displacements leading to the new equilibrium configuration, with a static method which is a discrete analog of elastoplastic finite element calculations in continuum mechanics. Such methods are seldom used (see, however, (Kishino et al. 2001)) in granular systems because they are more complicated and less versatile than the usual dynamical approaches: a stiffness matrix has to be rebuilt for each different contact list, and calculations are limited to the range of stability of a given contact network. As long as the contact structure is able to support the load, plastic strains in the sliding contacts remain contained by elastic strains in the non-sliding ones, and the static method is able to determine the sequence of configurations reached on, e.g., stepwise increasing $q$. This sequence is made of a continuous set of equilibrium states, and the system evolution is indeed quasistatic: we refer to such case 
as the strictly quasistatic régime. We checked, for series A samples, that static and dynamic calculations are in perfect agreement in such cases, as shown on fig. 6. This initial régime is the stability range of the initial configuration. The strains are then directly due to contact deformation - such strains will be termed of type $I$ in the sequel - and are inversely proportional to $\kappa$, while results are not sensitive to $\gamma$ (the static method ignores completely inertia and physical time). This range should not be regarded as an elastic domain, as the non-linearity of the curves on fig. 6 (the elasticity of contacts is linear) is due to contact losses and also to the gradual mobilization of friction. On reversing the $q$ increments, steeper slopes are observed. In the samples of fig. 6, the very steep parts of the staircase-shaped curves also correspond, as we checked, to stability intervals of some intermediate equilibrium configuration at higher $q$. Such intervals are separated by large strain steps, corresponding to rearrangements of the contact structure. Those occur when the accumulation of sliding contacts leads to an instability, and the ensuing motion is arrested by new contacts as interstices between neighbouring grains are closed. The resulting strain increments are hereafter referred to as type II strains. Their magnitude is related to the width of interstices between neighbouring grains. The system evolution, in that rearrangement régime, is, as shown previously, more sensitive to dynamical parameter $\gamma$. Equilibrium states do not form a continuum in configuration space, the system has to jump between two successive ones in a controlled deviator step test, or to flow nearby in a controlled strain rate test. The evolution can only be termed quasistatic in a wider sense if the statistical properties of trajectories in configuration space are independent, for slow enough motions, on dynamical parameters - which can be reasonably expected from the present study. The initial strictly quasi-static $q \leq q_{1}$ interval does not shrink, but appears rather to approach a finite limit (about $q_{1}=0.8$ here) as the sample size increases. Stress-strain curves depend on $K_{T} / K_{N}$ within this range, but, interestingly, $q_{1}$ does not (Combe 2001). In the rearrangement régime, in order to approach a smooth curve in the macroscopic limit (see fig. 2), it is necessary that the sizes of both the steep and the flat parts of the 'staircases' shrink to zero as the sample size increases. Type I and type II strains have very different amplitudes in A samples with $\kappa=10^{5}$ and $N \leq 4900$. It might in fact be expected that this clearcut distinction will get blurred at smaller stiffness parameter $\kappa$ (whence larger type I strains) or larger $N$ (as smaller type II strain increments can close contacts), and that the transition at $q_{1}$ will be fuzzier. Nevertheless, the system properties do strongly differ for $q<q_{1}$ and $q>q_{1}$, in two important respects. First, the slope of the stress-strain

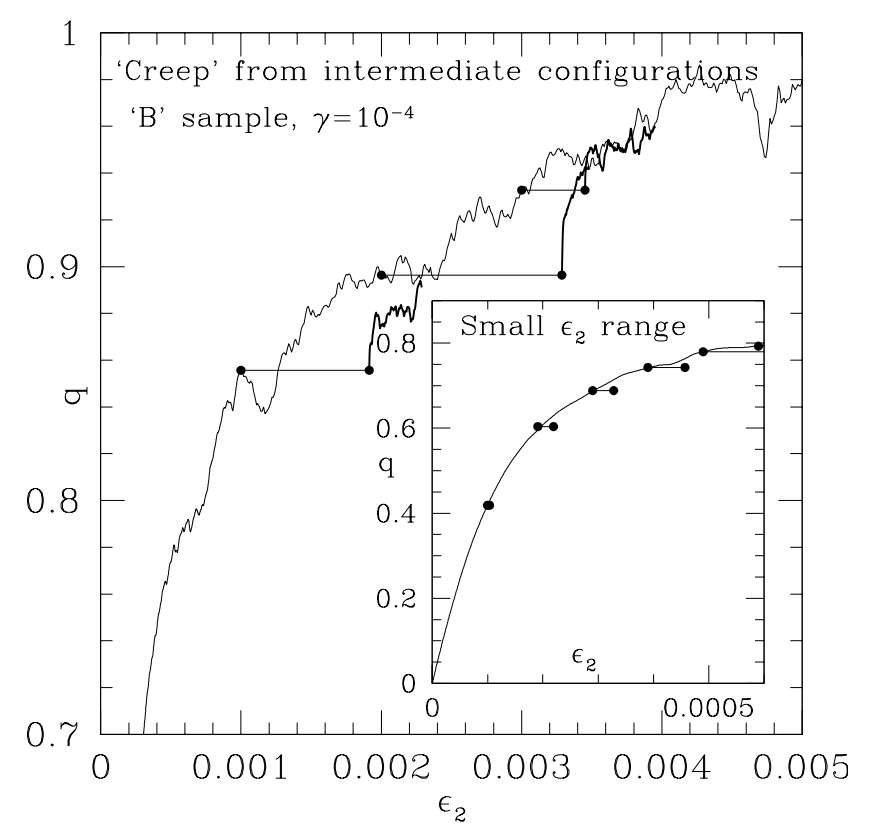

Figure 7: 'Creep tests', dots on main plot showing initial and final (equilibrium) states. Effect of resuming compression SRC way shown as thick lines. Inset: creep tests within strictly quasistatic range.

curve relates directly to the elasticity of the contacts in the type I strain dominated, strictly quasistatic case. The tangent at the origin on fig. 6 (smaller plot) is the Young modulus of the packing. Second, the amplitude of fluctuations, the distance to mechanical equilibrium, and the sensitivity to perturbations are much stronger in the rearrangement (type II strain dominated) régime. This is further illustrated by the following 'creep experiment' $:$ in a strain-rate controlled biaxial compression, at some arbitrary instant, shift to stress-controlled conditions and keep $q$ constant, until an equilibrium configuration is reached. Typical results of such tests are shown on fig. 7. As could be expected, much larger strain variations are observed during periods of creep in the rearrangement regime, as the initial states are farther from equilibrium. On resuming the constant strain rate test, the initial part of the curve is very steep, which is characteristic of a 'strictly quasistatic' interval. From an equilibrium state (devoid of sliding contacts), friction has to be mobilized again to produce the instabilities of the rearrangement régime. The dilatancy within those creep intervals is similar to the SRC one.

The 'creep tests' reveal different behaviours in the two deformation regimes in SRC tests. One might also probe the sensitivity to perturbations of intermediate equilibrium states obtained in SIC tests. We repeatedly applied on the grains constant external forces, each force component being randomly chosen between $-f_{0}$ and $f_{0}\left(f_{0}\right.$ is a small fraction of $\left.a P\right)$, until new, perturbed equilibria were reached. Such random load increments always tend to produce strains 


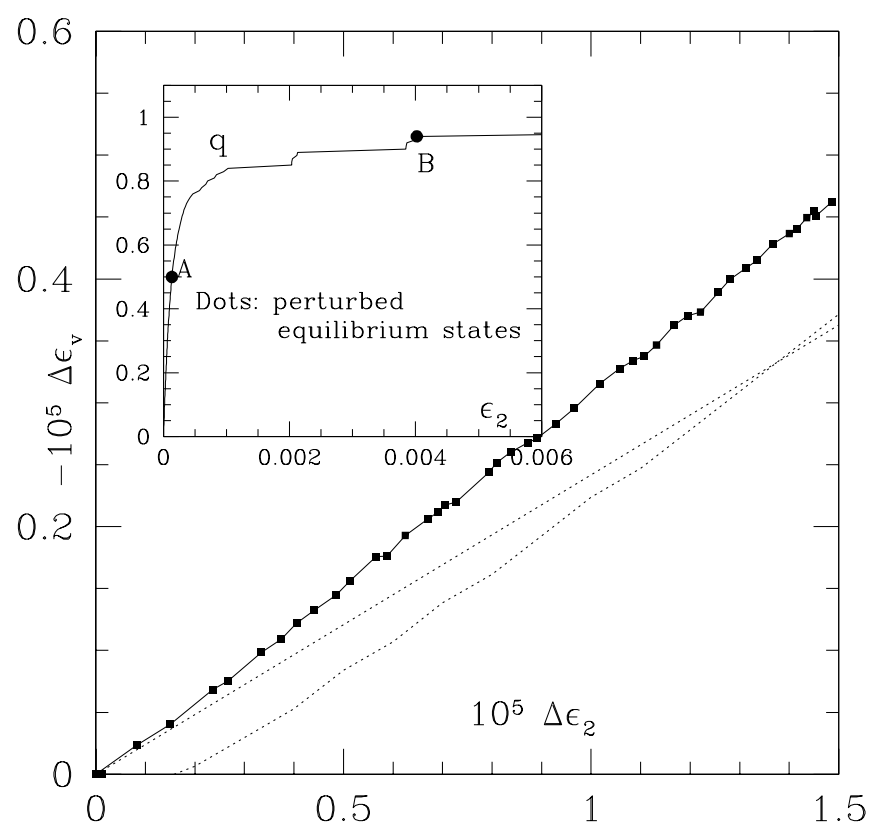

Figure 8: Effect of repeated random load $\left(f_{0} / a P=\right.$ $2.510^{-3}$ ) applied in states shown as big dots on the stressstrain curve in the inset: increments of $\epsilon_{v}$ vs. increments of $\epsilon_{2}$ on blown-up (by $10^{5}$ ) scale. The response of state $\mathrm{A}$ is concentrated near the origin, only the response of state $\mathrm{B}$ ( $q=0.94)$ is visible on this scale. Dotted lines: SIC and SRC dilatancy curves near point B, same sample.

in the same direction, as illustrated on fig. 8. Applied when $q=0.5$ within the strictly quasistatic range, such perturbations entail very small strain increments (hardly visible near the origin of the plot). Applied when $q=0.94$ as equilibrium states are much more unstable, they produce the series of strain increments plotted as connected dots, which tend to accumulate proportionnally, hence the nearly straight line, the slope of which is comparable to the dilatancy. The repeated application of small random perturbations thus entails some 'creep' phenomenon.

\section{COMPARISONS WITH EXPERIMENTS}

In spite of the many differences between the numerical models and the materials studied in the laboratory, such as sand, or even glass beads, some features of the simulation results can be compared in a qualitative or semi-quantitative way to experimental ones.

First, parameters $\kappa$ and $\gamma$ should be used to obtain robust estimations of orders of magnitude. In 3 dimensions, $\kappa$ should be defined as $K_{N} /(a P)$ in the case of linear elasticity in the contacts. $\kappa$ measures the normal elastic deflection in a contact, relatively to the grain diameter $a$, due to the typical contact force $P a^{2}$. In a Hertzian contact between spheres of diameter $a$, it is easy to show that $\kappa$ should be defined as $(E / P)^{2 / 3}$, where $E$ is the Young modulus of the grain material. This gives $\kappa \simeq 6000$ for glass beads un- der $P=10^{5} \mathrm{~Pa}$. (In 3D simulations, we could check that, given these definitions, the effect of $\kappa$ on the coordination number was similar to the 2D case, see also (Makse et al. 2000)). 'Real' materials with Hertz contacts under $P=10^{5} \mathrm{~Pa}$ are rather on the rigid side, but not quite in the rigid limit. Other contact laws might lead to even smaller stiffness parameters (e.g., $\kappa \sim(E / P)^{1 / 2}$ if $F_{N} \propto E h^{2}$, as for cone-shaped asperities).

An appropriate 3D definition of $\gamma$ is $\dot{\epsilon} \sqrt{\frac{m}{a P}}\left(\sqrt{\frac{m}{a P}}\right.$ is the time for a grain accelerated from rest by the typical force $a^{2} P$ to move on distance $a / 2$ ). Substituting typical values - a fraction of millimetre for $a$, $10^{-5} s^{-1}$ for $\dot{\epsilon}-$ this yields $\gamma$ values as small as $10^{-9}$ or $10^{-10}$. As calculations over $\epsilon=2 \%$ strain intervals with $\gamma=10^{-5}$ still require several days of c.p.u. time with 5000 stiff grains, real time scales of quasistatic laboratory tests are still beyond the reach of discrete numerical simulations. $\gamma$ dependences of numerical results can however be extrapolated to smaller values.

Although it is tempting, in view of the results illustrated on fig. 7 to refer to creep experiments (Matsushita et al. 1999; Di Benedetto and Tatsuoka 1997), as the aspects of the stress-strain curves are quite similar in several respects, this difference of time scales precludes a direct comparison. Moreover, the experimental $q-\epsilon$ curves do not depend on strain rate if it is constant (this corresponds to much smaller $\gamma$ values than simulations), and the creep deformation is extremely slow, often logarithmic in time (Di Prisco and Imposimato 1997). Unlike in the numerical case, it does not appear to stop as some equilibrium is reached. It might well be relevant, however, to discuss such experiments in terms of the sensitivity of the system to perturbations, which is likely to depend on whether contact networks resist load increments (strictly quasistatic case) or are prone to instabilities (rearrangement régime). The numerical tests discussed in connection with fig. 8 suggest a possible microscopic origin of such slow evolutions over long times: a small noise level, always present in experiments, could entail an accumulation of strain. Aging and creep phenomena can also be physically expected within one contact. Numerical simulations (devoid of such features) might help assessing the collective aspects of the packing response.

Our simulations can also be likened to experimental observations about the very small strain elastic behaviour of granular systems (Di Benedetto et al. 1999). Recent developments of precision apparati enabled measurements of strains in the $10^{-5}$ range. To obtain elastic moduli, small stress cycles are superimposed on a constant loading, producing cyclic strains on top of a systematic drift which, on increasing the number of cycles, gradually slows down and becomes analogous to the one observed in creep tests. The av- 
erage slope of a cycle on a stress-strain plot, once the effect of the drift is negligible, can be interpreted as an elastic modulus (there remaining some small dissipation). Those small strain increment elastic constants agree with the ones deduced from acoustic wave velocities. From our simulations, it transpires that the incremental stress-strain dependence might express a genuinely elastic behaviour (supplemented by some plastic dissipation which vanishes in the limit of small stress increments) in the strictly quasistatic régime. Elastic moduli are then related to the stiffness of the contacts. The width of strictly quasistatic strain intervals are of the order of $q_{1} / \kappa-q_{1}$ being their width in terms of stress ratio. Taking into account that $q_{1}$ is exceptionally large for the initial small-strain régime if our extremely dense and well coordinated systems, and the value $\kappa \sim 6000$ estimated above for glass beads, one does obtain the right order of magnitude $\left(\leq 10^{-4}\right)$ for the very small strain elastic domain. Moreover, the procedure by which these moduli are measured can be interpreted as the preparation, either left to random perturbations or forced by cyclic load increments, of a better stabilized state for which the contact network is able to resist small, but finite stress increments (just like the stiffly responding equilibrium states of fig. 7).

\section{CONCLUSIONS AND PERSPECTIVES}

Despite their limitations (due to the simplicity of the contact model, and the inaccessibility of long time scales), the numerical simulation results presented here enable some investigation of the microscopic origins of many features of experimentally observed behaviours. The definition of reduced dimensionles parameters $(\kappa$ and $\gamma)$ provides a framework in which many experimental and numerical studies can be discussed in common terms. Due to the small size of numerical samples, constitutive laws have to be approached via statistical analyses. Most importantly, the distinction between two different origins of strain and two deformation régimes allows us some interpretations of very small strain (tangential) elasticity and slow deformation (creep) under constant load, in terms of the system sensitivity to perturbations.

This work should be pursued in three directions. First, it is desirable to extend the existing approach to more 'realistic' models, so that more quantitative comparisons with experiments will be possible (our $3 \mathrm{D}$ results on spheres - an obvious step in this direction, were not presented here for lack of space). Secondly, the importance of the initial state and of the sample preparation procedure calls for systematic studies (unlike for quasistatic monotonous compression tests, experimental knowledge is not expressed as well established laws for such processes). And, finally, the joint use of dynamic and static methods, which agree remarkably in strictly quasistatic domains (fig. 6) opens avenues to explore fundamental issues, such as elastoplastic contact network stability and rearrangements, in some microscopic detail.

\section{REFERENCES}

Benahmed, N. (2001). Liquéfaction des sables. Ph. D. thesis, École Nationale des Ponts et Chaussées, Marne-la-Vallée.

Combe, G. (2001). Origines géométrique du comportement quasi-statique des assemblages granulaires. Ph. D. thesis, École Nationale des Ponts et Chaussées, Marne-la-Vallée.

Cundall, P. A. and O. D. L. Strack (1979). A discrete numerical model for granular assemblies. Géotechnique 29(1), 47-65.

Di Benedetto, H., H. Geoffroy, and C. Sauzéat (1999). Sand behaviour in very small to medium strain domains. See Jamiolkowski (1999), pp. 89-96.

Di Benedetto, H. and F. Tatsuoka (1997). Small strain behaviour of geomaterials: modelling of strain rate effects. Soils and Foundations 37(2), 127-138.

Di Prisco, C. and S. Imposimato (1997). Experimental analysis and theorical interpretation of triaxial load controlled loose sand specimen collapses. Mechanics of cohesive-frictional materials 2, 93-120.

Jamiolkowski, M. Lancellotta, R., and Lo Presti, D. (Eds.) (1999). Pre-failure deformation characteristics of geomaterials, Rotterdam. Balkema.

Kishino, Y. (Ed.) (2001). Powders and Grains 2001, Lisse. Swets \& Zeitlinger.

Kishino, Y., H. Akaizawa, and K. Kaneko (2001). On the plastic flow of granular materials. See Kishino (2001), pp. 199-203.

Kuhn, M. R. (1999). Structured deformation in granular materials. Mechanics of materials 31, 407-429.

Makse, H., D. Johnson, and L. Schwartz (2000). Packing of compressible granular materials. Physical Review Letters 84(18), 4160-4163.

Matsushita, M., F. Tatsuoka, J. Koseki, B. Cazacliu, H. Di Benedetto, and S. J. M. Yasin (1999). Time effects on the pre-peak deformation properties of sands. See Jamiolkowski (1999), pp. 681-689.

Roux, J.-N. (2000). Geometric origin of mechanical properties of granular materials. Physical Review E 61(6), 6802-6836.

Roux, J.-N. and G. Combe (2002). Quasistatic rheology and the origins of strain. C. R. Académie des Sciences (Physique) 3, 131-140.

Silbert, L. E., D. Ertas, G. S. Grest, T. C. Halsey, and D. Levine (2001). Geometry of frictionless and frictional sphere packings. Phys.Rev. E 64, 051302.

Wood, D. M. (1990). Soil Behaviour and Critical State Soil Mechanics. Cambridge University Press. 